\title{
Entwicklung, Einsatz und Evaluation eines Tools für digitales Ideenmanagement. Ein Fallbeispiel
}

\author{
Anna T. Röltgen ${ }^{1}$ (D) - Valeria Bernardy ${ }^{1}$ (D) $\cdot$ Rebecca Müller $^{1}$ (D) · Conny H. Antoni' ${ }^{1}$ \\ Online publiziert: 28. Januar 2020 \\ (c) Der/die Autor(en) 2020
}

\section{Zusammenfassung}

Dieser Beitrag der Zeitschrift Gruppe. Interaktion. Organisation. (GIO) analysiert die Entwicklung eines Tools für digitales Ideenmanagement (IdeaCheck), und dessen Implementierung anhand eines Fallbeispiels aus einem mittelständischen deutschen IT-Unternehmen. Ziel ist es, das inhaltliche Konzept von IdeaCheck aufbauend auf psychologischen Theorien zu erläutern und Erfahrungen mit dem Tool bei der ersten Implementierung zu berichten sowie Möglichkeiten für die Anwendung und Weiterentwicklung von dem IdeaCheck zu diskutieren. Dabei werden anhand eines Fallbeispiels zunächst die Herausforderungen beschrieben, die durch die zunehmende Digitalisierung der Zusammenarbeit für das Ideenmanagement entstehen. Anschließend wird der Aufbau des Tools beschrieben und dargestellt, welche Ziele mit dem IdeaCheck erreicht werden sollen. Der erste Einsatz des IdeaCheck in einem Unternehmen wurde formativ evaluiert. Die dabei gewonnenen Erkenntnisse werden reflektiert und Perspektiven für die weitere Anwendung und Weiterentwicklung des Tools aufgezeigt.

Schlüsselwörter Ideenmanagement · Innovation · Digitale Zusammenarbeit · Formative Evaluation

\section{Development, implementation and evaluation of a digital idea management system. A case analysis}

\begin{abstract}
This article in the journal Gruppe. Interaktion. Organisation. (GIO) analyses the development and implementation of a digital idea management tool (IdeaCheck) based on a case study of a medium-sized German IT company. The aim is to explain the concept of IdeaCheck with regard to underlying psychological theories and to reflect critically the experiences with the tool during its first implementation. Based on these findings, potentials for application and future development of IdeaCheck will be discussed. Within the case study, we first describe the challenges that digital collaboration poses for idea management. Subsequently, the structure of IdeaCheck and its objectives are described. The first use of IdeaCheck in a company was evaluated formatively. These results are reflected and perspectives for the further application and development of the tool are discussed.
\end{abstract}

Keywords Idea management $\cdot$ Innovation $\cdot$ Digital collaboration $\cdot$ Formative evaluation

\section{Einleitung: Ein Fallbeispiel}

Das Unternehmen IT entwickelt innovative, qualitativ hochwertige kundenspezifische IT-Lösungen in einem kompetitiven Marktumfeld. Bislang werden Produkt- und Prozess-

Anna T. Röltgen, M.Sc.

roeltgen@uni-trier.de

1 Abteilung für Arbeits-, Betriebs- und Organisationspsychologie, Universität Trier, Universitätsring 15, 54296 Trier, Deutschland ideen (z.B. Neu- und Weiterentwicklungen von Hard- oder Software im IT-Bereich), die im Rahmen kundenspezifischer Projekte entstehen, aber dort nicht umgesetzt werden können, häufig nicht festgehalten und gehen verloren. Wenn Mitarbeiter und Mitarbeiterinnen (MA) eine Idee einbringen wollen, müssen sie sich an ihre Vorgesetzten wenden und hierfür einen günstigen Moment abpassen. Ist die Idee überzeugend, wird sie an andere Entscheidungsbefugte in der Hierarchie weitergeleitet. Es gab in der Vergangenheit mehrfach den Versuch, alle MA in den Innovationsprozess einzubinden. Diese scheiterten an fehlenden zeitlichen Ressourcen, fehlender oder stark zeitverzögerter Rückmeldung 
und der Intransparenz des hierarchischen Entscheidungsprozesses.

Aufgrund neuer Standorte, der vermehrten Arbeit beim Kunden vor Ort und Homeoffice-Regelungen, nimmt der persönliche Kontakt ab und der Anteil digitaler Kommunikation zu: Dinge, die früher persönlich besprochen wurden, werden jetzt per Email, Telefon oder anderen digitalen Medien geklärt. Der Austausch beschränkt sich angesichts des zunehmenden Zeitdrucks auf das Wesentliche und Aufgabenrelevante. Der Vertrauensaufbau über digitale Medien fällt MA schwer.

Dementsprechend hoch ist der Bedarf nach einem effizienten Innovations- und Ideenmanagement: Das Management möchte die IT-MA stärker in den Innovationsprozess einbinden - aber wie und welche Rahmenbedingungen müssen gegeben sein, damit sich die MA einbringen (können und wollen)?

\section{Digitalisierung und Innovation}

Die im Fallbeispiel beschriebenen Herausforderungen werden auch in der Literatur diskutiert. Digitalisierung und Innovation bedingen sich gegenseitig. Innovative technische Entwicklungen ermöglichen und unterstützen die räumlich und zeitlich verteilte Zusammenarbeit (Akin und Rumpf 2013). Dazu gehören Entwicklungen in der Hardware wie Computer und mobile Endgeräte (z.B. Laptops, Tablets und Smartphones) und kollaborative Software, die das gemeinsame Arbeiten an Dokumenten und den Zugriff auf Informationen ermöglichen (Anders 2016; Bafoutsou und Mentzas 2002). Durch die Digitalisierung sind mehr Informationen schneller verfügbar (Antoni und Ellwart 2017): der schnelle Zugang zu vielfältigen (nichtredundanten) Informationen hat zwar einerseits einen Einfluss auf die Innovationsfähigkeit und die Entwicklung von Innovationsideen (Ruef 2002), andererseits führt die Beschleunigung der Arbeitsprozesse zu mehr Zeitdruck, Informationsüberlastung und Stress (Eppler und Mengis 2004). Bei steigendem Stress fokussieren sich MA auf ihre primären (Arbeits-)Aufgaben zu Lasten von sekundären Aufgaben (Hockey 1997). Indes erschwert der vermehrte Einsatz digitaler Kanäle in der Kommunikation den Aufbau von Vertrauen untereinander (Breuer et al. 2017; Handke und Kauffeld 2019). Vertrauen und psychologische Sicherheit sind jedoch die Basis des kreativen Austauschs und damit ein entscheidender Faktor im Innovationsprozess (Edmondson und Lei 2014). Der fehlende persönliche Austausch erschwert somit den Aufbau von Vertrauen und senkt dadurch letztlich die Bereitschaft Innovationsideen für Produkte und Prozesse einzubringen. Gleichzeitig steigt jedoch der Stellenwert von Innovationen bei klein- und mittelständischen Unternehmen (KMU) einhergehend mit der schnellen Entwicklung der Märkte (Spielkamp und Rammer 2006; Rammer et al. 2016). Innovationen sind ein zentraler Faktor, um Umsatz, Gewinn und Beschäftigung zu steigern und die Überlebensfähigkeit von Unternehmen zu sichern (Möslein 2009). In der Konsequenz stehen KMUs unter einem regelrechten Innovationsdruck.

Wie das Fallbeispiel verdeutlicht, fehlen Vertrauen, zeitliche und kognitive Ressourcen, um neue Ideen neben dem Tagesgeschäft auszuarbeiten, innerhalb des Unternehmens zu kommunizieren und zu unterstützen, mit der Gefahr, dass diese verloren gehen. Die Unternehmen stehen damit vor dem Dilemma, dass die Digitalisierung das Potential steigert, Innovationsideen zu entwickeln, aber gleichzeitig die Kapazitäten reduziert, diese Ideen festzuhalten, auszuformulieren und innerhalb des Unternehmens zu kommunizieren.

In diesem Beitrag wollen wir beleuchten, inwieweit die Einführung eines digitalen Tools zum Ideenmanagement (IdeaCheck) diesen im Fallbeispiel und in der Literatur beschriebenen Herausforderungen begegnet und die Innovationsfähigkeit von Unternehmen fördern kann, welche Anforderungen sich für den Aufbau des Tools ergeben und welche Rahmenbedingungen einhergehend mit der Einführung Beachtung finden sollten.

\subsection{Anforderungen an ein digitales Ideenmanagement}

Aus dem Fallbeispiel und bisheriger Literatur ergeben sich übereinstimmend Anforderungen an das digitale Ideenmanagement. Bisherigen Ansätzen des Ideenmanagements, wie dem betrieblichen Vorschlagswesen (BVW), mangelt es an der Transparenz und Agilität, die im digitalen Zeitalter an Prozessabläufe und an die Mitbestimmungskultur gestellt werden (Hossain et al. 2009). Seit langem wird kritisiert, dass intransparente und langwierige Beurteilungsverfahren dazu führen, dass der Großteil der Vorschläge nur von einem kleinen Teil der MA gemacht werden (Antoni 1987). Es herrscht oft Unklarheit darüber, anhand welcher Kriterien über die Umsetzung der Idee entschieden wird und ob, in welchem Zeitraum und in welcher Form Feedback gegeben wird (von Bismarck 2000). Der Ideenmanagement- und Innovationsprozess erscheint dadurch intransparent und Innovationsideen werden nicht kommuniziert, zumal das Einbringen von Ideen eine freiwillige Leistung der MA ist (Frese et al. 1996). MA und -Führungskräfte aus dem Fallbeispiel (nachfolgend IT-MA genannt) berichteten ebenfalls von Versuchen, den Innovationsprozess nach innen zu öffnen und MA stärker miteinzubeziehen, welcher aufgrund mangelnder Transparenz gescheitert ist.

Der zweite Kritikpunkt, fehlende Agilität, bezieht sich unter anderem auf die Zeitkomponente: Ideenmanagement 
muss analog des iterativen Ansatzes an sich verändernde Bedingungen anpassbar sein und eine schnelle Umsetzungsentscheidung ermöglichen (Garcia und Calantone 2002). IT-MA berichteten von fehlender und zeitverzögerter Rückmeldung zu eingebrachten Ideen. Damit sei die Motivation der MA gesunken, erneut Ideen einzubringen. Agiles Ideenmanagement bedeutet, dass kurze Feedbackschleifen integriert werden und der Vergleich einzelner (Lösungs-)Ideen ermöglicht wird (Highsmith 2009). Entsprechend besteht Bedarf nach einem effizienten Ideenmanagement, das die digitale Abbildung des Prozesses von der Idee bis zur Umsetzungsentscheidung erlaubt, um das Motivationspotential aller MA zu aktivieren und eine zügige, teilautomatisierte und transparente Bewertungsund Umsetzungsentscheidung entsprechend der aus der Unternehmensstrategie abgeleiteten Bewertungskriterien zu gewährleisten (Spath et al. 2010).

Durch das hier vorgestellte Tool für digitales Ideenmanagement IdeaCheck soll, entsprechend den Empfehlungen in der Literatur (Abu El-Ella et al. 2013), der Innovationsprozess nach innen über die Grenzen von Abteilungen hinweg geöffnet und die Kollaboration gefördert werden. Dadurch sollen MA, die nicht zu den Kerninnovatoren im Unternehmen gehören, zu Akteuren bei der Ideengenerierung, -weiterentwicklung und -umsetzung und damit Teil des gesamten Produktentstehungsprozesses werden (Reichwald 2010).

Dabei ergeben sich für die Phasen Ideengenerierung und -implementierung Anforderungen auf unterschiedlichen Ebenen (Hammond et al. 2011). Damit MA Ideen generieren, sind motivationale Prozesse entscheidend. Insbesondere die Faktoren Wertschätzung und psychologische Sicherheit fördern für die Ideengenerierung entscheidende Kreativität (Amabile und Pratt 2016; Edmondson und Lei 2014). Für die Umsetzungsentscheidung und Implementierung spielen hingegen Kontextfaktoren wie Machbarkeit, finanzielle und zeitliche Ressourcen eine Rolle. Diese Kontextfaktoren sowie damit verbundenen Ressourcen unterscheiden sich zwischen Unternehmen. Deshalb soll das Tool eine individuelle Anpassung an das Unternehmen und dessen (Innovations-)Strategie ermöglichen und agile Arbeitsmethoden unterstützen.

Mit dem im Rahmen des BMBF-geförderten Projektes vLead entwickelten Tool IdeaCheck sollen vier Ziele erreicht werden, die sich aus mitarbeiter- und unternehmensseitigen Anforderungen des Fallbeispiels ergeben: (1) Der Innovationsprozess soll transparenter werden (Transparenz), (2) Ideen sollen nicht verloren gehen (Ideenspeicher), (3) die Umsetzungsentscheidung soll für alle Nutzende des Tools nachvollziehbar (Nachvollziehbarkeit) und (4) effektiv sein (Effektivität). Effektivität zeichnet sich in diesem Fall dadurch aus, dass Produkt- und Prozessideen weiterverfolgt werden, die sowohl erfolgsversprechend als auch mit den vorhandenen zeitlichen und finanziellen Ressourcen umsetzbar sind. Dadurch soll letztlich die Motivation der MA gesteigert werden, Ideen ins Unternehmen einzubringen. IdeaCheck bietet gegenüber vergleichbaren Systemen den Vorteil, dass die Struktur an die Bedürfnisse der KMUs angepasst werden kann. Dies geschieht über die individuelle Konfiguration des Ideenbewertungsprozesses und der Zuweisung verschiedener Rollen im Tool. Darüber hinaus steht das Tool während der Projektlaufzeit für KMUs kostenlos zur Verfügung.

\section{IdeaCheck: Aufbau und Beschreibung}

Entsprechend den oben beschriebenen Anforderungen wurde der IdeaCheck wie folgt gestaltet: Das Tool orientiert sich an der Stage-Gate-Struktur der Produktentwicklung (Cooper 2014). Stages sind die Stufen, die eine Idee durchlaufen muss, bis sie angenommen wird. Die Gates beschreiben Kriterien, die eine Idee erfüllen muss, um von einer Stufe in die nächste zu kommen. Die jeweiligen Bewertungskriterien werden in einer Scorecard (Friedrich 2014) festgehalten (Abb. 1).

Um eine unternehmensspezifische Anpassung zu ermöglichen, können sowohl die Stufenanzahl als auch die relevanten Kriterien für die Umsetzungsentscheidung verändert werden. Zusätzlich bietet IdeaCheck die Möglichkeit, Kategorien anzulegen. Diese können beispielsweise Produkte oder Unternehmensbereiche (z.B. Entwicklung) repräsentieren. Für jede Kategorie werden Stages und Gates konfiguriert. Dementsprechend können Ideen derselben Kategorie anhand der gleichen Kriterien (z. B. strategische Bedeutung) bewertet werden. Ideen einer Kategorie durchlaufen die gleiche Anzahl an Schritten, bis eine Umsetzungsentscheidung getroffen wird. Darüber hinaus können Bewertungskriterien unternehmens- oder bereichsspezifisch unterschiedlich gewichtet werden, um die jeweiligen Unternehmensziele abzubilden. Dementsprechend können die gleichen Kriterien an unterschiedlichen Gates verwendet und je nach Wichtigkeit und Entscheidungsrelevanz gewichtet werden. Dieser Aufbau soll einen transparenten, verständlichen und nachvollziehbaren Entscheidungsprozess gewährleisten.

Insgesamt bietet das online Tool IdeaCheck ein zentrales digitales Sammelbecken für Innovationsideen und alle MA haben die Gelegenheit, Ideen einzubringen. Nach dem Anlegen einer Idee, kann diese als Entwurf gespeichert werden. In diesem Fall kann kein anderer Nutzender die Idee einsehen und MA haben die Möglichkeit, eine Idee schnell festzuhalten und zu einem späteren Zeitpunkt so zu vervollständigen, dass sie für andere verständlich dargestellt ist. Wird eine Idee anschließend veröffentlicht, ist sie für alle Nutzenden des Tools einsehbar. 


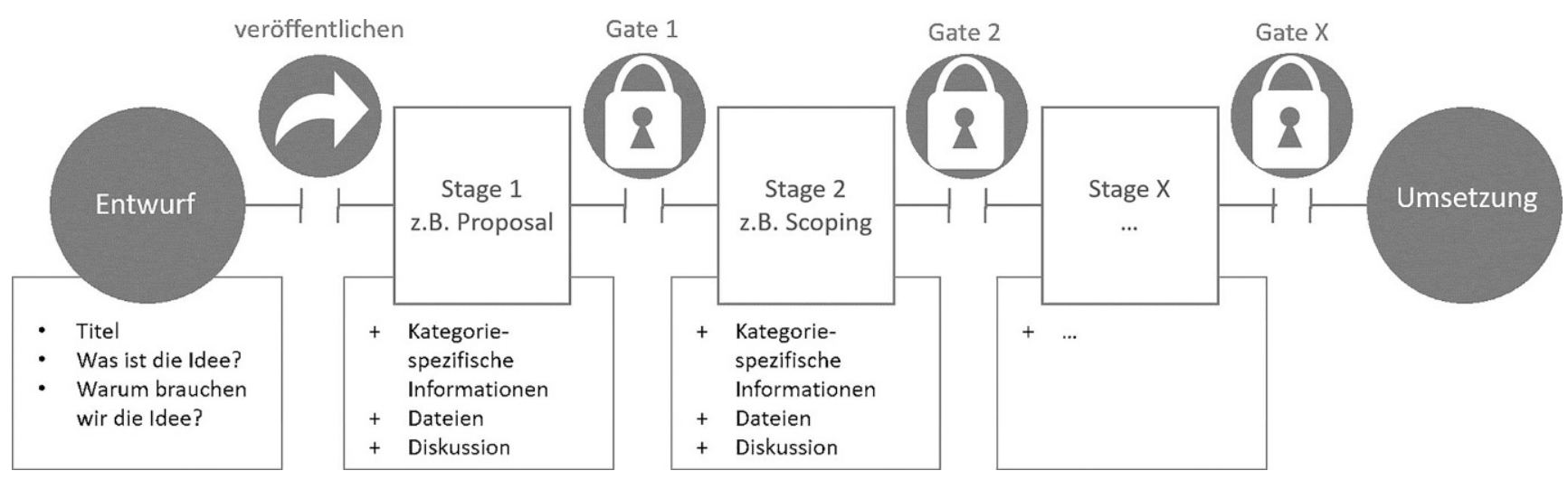

Abb. 1 Schematische Abbildung des Ideenbewertungsprozesses in IdeaCheck als Stage-Gate-Struktur

Nach der Veröffentlichung einer Idee kann diese zur Bewertung freigegeben werden. In diesem Fall bewerten festgelegte Nutzende die Idee anhand einer Punktevergabe in der Scorecard. Diese festgelegten Nutzenden sind Mitglieder des Entscheidungsgremiums und umfassen zumeist Führungskräfte mit entsprechenden Kenntnissen und Befugnissen. Wie viele und welche MA einem Entscheidungsgremium angehören, kann im Tool unternehmensspezifisch konfiguriert werden. Nachdem alle Mitglieder des Gremiums die Idee bewertet haben, wird diese an die Person zurückgemeldet, welche die Idee eingereicht hat. Mitglieder des Gremiums haben die Möglichkeit, über das Tool Feedback zu geben und ihre Entscheidung zu begründen. Erreicht eine Idee ausreichend Punkte, geht sie nach der Bewertung in die nächste Stage über. Wird die benötigte Punktzahl bei der Bewertung nicht erreicht, verbleibt die Idee in der aktuellen Stage. In diesem Fall können MA auf Grundlage des Feedbacks entscheiden, ob sie an der Idee weiterarbeiten und diese anschließend erneut zur Bewertung freigeben oder die Idee nicht weiterverfolgen. Mitglieder des Gremiums werden dazu angehalten, eine entsprechende Empfehlung im Rahmen des Feedbacks zu hinterlegen.

Verschiedene Funktionen ermöglichen es, sich an den Ideen anderer zu beteiligen. Man kann sich in Form von Diskussionsbeiträgen einbringen, eine Idee mit der eigenen Expertise unterstützen und einer Idee folgen und damit über Neuigkeiten auf dem Laufenden bleiben. Im Tool ist für jeden Nutzenden ein Profil mit Informationen zur Abteilungszugehörigkeit hinterlegt, welches zusätzlich um Information zu Expertise und Interessen ergänzt werden kann. Diese Funktionen ermöglichen es MA aktiv nach Unterstützenden mit bestimmten Kenntnissen zu suchen, um die Idee weiterzuentwickeln.

\section{Durchführung, Ergebnisse und Erkenntnisse der formativen Evaluation}

Im Rahmen einer formativen Evaluation wurde die Betaversion des IdeaChecks unterschiedlichen Personengruppen vorgestellt. Bereits in der Vorbereitung der formativen Evaluation zeigte sich, dass das hohe Individualisierungs- und Anpassungspotential des Tools an die Unternehmensstruktur mit einem hohen Konfigurationsaufwand einhergeht. Damit der Innovationsprozess im Tool abgebildet werden kann, muss zunächst seine Struktur festgelegt werden. Dazu gehören Anzahl der Kategorien, Definition von Anzahl und Inhalt der Entscheidungsschritte (Stages und Gates), die Festlegung von Bewertungskriterien und die Zusammensetzung der Entscheidungsgremien. Die Umsetzungsentscheidung von Innovationsideen können lediglich von Personen getroffen werden, die eine Berechtigung zur Freigabe von finanziellen, personellen und zeitlichen Ressourcen haben (Hauschildt et al. 2016). Bei KMUs liegt diese Aufgabe im Besonderen bei der Geschäftsführung (Spielkamp und Volkmann 2005), dies zeigt sich auch in unserem Fallbeispiel. Eine weitere notwendige Überlegung bezieht sich darauf, in welchem Abstand über die Umsetzung von Innovationsideen entschieden wird. Das Treffen einer fundierten Entscheidung und die im Rahmen der Entwicklung von IdeaCheck geforderten Ausführlichkeit des Feedbacks sei laut Aussage der IT-MA in der Praxis nur schwer mit einer zeitnahen Rückmeldung vereinbar, die aus motivationaler Sicht und entsprechend agiler Organisationsprinzipien wünschenswert wäre.

Die Betaversion lief zum Evaluationszeitpunkt über das Intranet des Unternehmens IT und wird im Rahmen der späteren Projektphase zur Überprüfung der Transferfähigkeit anderen Unternehmen online zur Verfügung gestellt. Ziel der formativen Evaluation war es, das Tool in Hinblick auf Akzeptanz, Benutzung und Konformität zu den Unternehmenszielen Transparenz, Ideenspeicher und Nachvollziehbarkeit zu evaluieren. Das Bewertungskonzept ist ange- 
lehnt an das Technologie-Akzeptanzmodell (Theory of Acceptance and Use of Technology, UTAUT, Venkatesh et al. 2003), welches zur Erklärung und Vorhersage individuellen Nutzungsverhaltens entwickelt wurde (Peris und Nüttgens 2011).

Es wurde ein gemischt qualitativ-quantitatives Vorgehen gewählt (Hegner 2003). Sechs MA von IT wurden während der Nutzung des Tools beobachtet, während sie a priori formulierte Testaufgaben durchführten, bei denen es darum ging, eine Innovationsidee im Tool einzutragen. Die MA wurden gebeten, ihre Gedanken zu Aufbau, Oberfläche und Funktion des Tools sowie Überlegungen zum Vorgehen bei der Aufgabenbearbeitung laut auszusprechen (Thinking Aloud Methode nach Jørgensen 1990). Zu Beginn der Beobachtung wurde den MA die Möglichkeit gegeben, das Tool frei zu explorieren. Dabei stand ihnen eine Hilfefunktion zur Verfügung, die die wichtigsten Eigenschaften und Funktionen des Tools aufzeigt. Nach der Aufgabenbearbeitung wurden die MA mit einem leitfadengestützten Interview zum Tool befragt. Darüber hinaus wurde eine Expertenbefragung (interessierte Personen aus Praxis und Verbundprojekten) zum IdeaCheck im Rahmen mehrerer Projektveranstaltungen durchgeführt, denen das Tool vorgestellt wurde. Feedback wurde im Rahmen von Kartenabfragen und Diskussionsrunden sowie mithilfe eines standardisierten Fragebogens eingeholt. Mit insgesamt 20 Items wurden verschiedene Aspekten des UTAUT Modells (effort expectancy, facilitating conditions, behavioral intention to use the system) sowie die Ziele Transparenz, Ideenspeicher, Nachvollziehbarkeit und Effektivität auf einer Antwortskala von 1 (stimme nicht zu) bis 5 (stimme zu) erfasst $(N=14)$. Da ihnen das Tool nur vorgestellt wurde, sie es aber nicht selbst genutzt haben, sind die Ergebnisse noch mit Vorbehalt zu betrachten. Eine standardisierte Befragung von IT-MA die das Tool nutzen, ist für die anstehende Pilotphase geplant, um die hier berichteten Ergebnisse abzusichern.

Bei der Beobachtung im Umgang mit dem Tool berichteten die MA im Hinblick auf Benutzbarkeit, dass sie die Benutzeroberfläche positiv wahrnehmen und das Tool im Allgemeinen intuitiv bedienbar sei. In der Expertenbefragung wurde die Benutzbarkeit des Tools hoch eingeschätzt (effort expectancy, $M=4,49, S D=0,41$ ). Die Hilfefunktion wurde in der Beobachtung nicht von allen MA genutzt, in der Befragung allerdings als hilfreich und inhaltlich verständlich bewertet (facilitating conditions, $M=4,55, S D=0,47$ ). Es wurde mehrfach berichtet, es seien bei der Eingabe der Idee zu viele Informationen abgefragt worden. Bei der Ersteingabe sei die Idee nicht soweit durchdacht gewesen, dass alle geforderten Pflichtfelder ausgefüllt werden konnten. Aus diesem Grund sei die Motivation gesunken, die Idee einzutragen. Welche und wie viele Informationen bei jedem Prozessschritt gefordert sind, ist konfigurierbar. Dementsprechend wurde im Anschluss an die formative Evaluation, die Zahl der vorgegebenen und vorstrukturierten Pflichtfelder bei der Ersteingabe der Idee reduziert. Dies ermöglicht den MA, die Idee in eigenen Worten festzuhalten (Titel, Beschreibung und Ursprung der Idee) und Ideen im Tool zu skizzieren, die noch nicht ausgereift sind. Die Idee kann somit als Ideenskizze im Tool veröffentlicht werden. Bevor die Idee allerdings zur Bewertung freigegeben werden kann, müssen weitere Informationen ergänzt werden. Dieses Beispiel verdeutlicht das Dilemma bei der Vorgabe von Strukturen: klare Strukturen sollen einerseits Unsicherheiten reduzieren und eine für die Geschäftsführung nachvollziehbare Darstellung der Ideen ermöglichen. Andererseits reduzieren Strukturen die Motivation der MA, sich einzubringen, wenn Aufwand und Nutzen (subjektiv) nicht mehr in angemessenem Verhältnis zueinanderstehen.

In Hinblick auf die Ziele Transparenz, Ideenspeicher und Nachvollziehbarkeit der Umsetzungsentscheidung zeigten sich die folgenden Ergebnisse. Die MA berichteten, der Innovationsprozess sei im Arbeitsalltag wenig sichtbar und sie erhofften sich von der Einführung des Tools im Unternehmen, dass das Thema Innovation stärker in den Arbeitsalltag integriert würde. Weiterhin wird dem Tool das Potential zugesprochen, den Innovationsprozess transparenter und für alle einsehbar zu machen. Die Strukturierung der Ideen wurde von den IT-MA positiv bewertet, die den bisherigen Innovationsprozess als eher unübersichtlichen Ideenpool wahrgenommen haben. Die Transparenz werde aus Sicht der MA ebenfalls durch die Funktionen Unterstützen und Idee folgen gefördert. Die Idee hinter der Funktion Unterstützen ist es, MA die Möglichkeit zu bieten, ihre Expertise einzusetzen und dadurch aktiv zur Weiterentwicklung einer Idee beizutragen. Durch diese Funktion können MA ebenfalls signalisieren, dass sie eine Idee gut finden. Die Funktion Idee folgen soll es MA ermöglichen, über den Fortschritt einer Idee auf dem Laufenden zu bleiben. MA bekommen nach Aktivierung dieser Funktion angezeigt, wenn die Idee in eine neue Phase übergeht oder von einem Gremium bewertet wurde. Unabhängig dieser Funktionen haben alle MA die Möglichkeit, Diskussionsbeiträge zu hinterlassen, was die IT-MA ebenfalls als positiv bewerteten. Dass der Innovationsprozess mithilfe von IdeaCheck transparent gestaltet werden könne, wird von den Ergebnissen der Expertenbefragung gestützt $(M=4,46, S D=0,52)$.

Darüber hinaus sprachen die IT-MA dem Tool das Potential zu, als übersichtlicher Ideenspeicher fungieren zu können. Dies würde insbesondere durch die Kategorienzuordnung ermöglicht. Diese Annahme wird von den Ergebnissen der Expertenbefragung gestützt $(M=4,50, S D=0,52)$. Darüber hinaus wird es von den IT-MA als wichtig erachtet, Ideen nicht nur mündlich zu kommunizieren, sondern diese auch schriftlich in standardisierter Form festzuhalten. Die entsprechende Funktion, Ideen abzubilden, zu veröffentlichen und anschließend strukturiert weiterzuentwi- 
ckeln, wurde von den IT-MA und in den Diskussionsrunden positiv bewertet. Dabei würden alle Ideen nicht nur für das Unternehmen, sondern auch für die MA selbst dokumentiert und gerieten nicht in Vergessenheit. Insbesondere, wenn eine Idee zum aktuellen Zeitpunkt nicht umgesetzt werden könne und zu einem späteren Zeitpunkt nochmal aufgegriffen werden solle, biete das Tool Unterstützung dabei, Ideen nachvollziehbar zu dokumentieren.

Die Umsetzungsentscheidung so darzulegen, dass sie für alle MA nachvollziehbar ist, stellt eine weitere Herausforderung dar. Die Darstellung der Kriterien und der Rückmeldung, wie diese im Einzelnen beurteilt wurden, wurde in der Expertenbefragung als Schritt in die richtige Richtung bewertet und als nachvollziehbar wahrgenommen $(M=4,15, S D=0,90)$. In der Expertenbefragung wurde zusätzlich angemerkt, dass die Gremienmitglieder durch die Bewertungskriterien sowohl bei der fundierten Entscheidungsfindung als auch bei der Begründung ihrer Entscheidung unterstützt werden würden. Es werde darüber hinaus als wichtig erachtet, dass MA die Chance haben, ihre Idee nachzubearbeiten und die Anmerkungen aus der Bewertung umzusetzen.

Die befragten IT-MA berichteten, einen strukturierten Entscheidungsprozess im Unternehmen zu etablieren sei zeitaufwendig und erfordere kontinuierliche Anpassungen. Entscheidungen, eine Idee weiterzuverfolgen und umzusetzen, könne nicht ohne weiteres automatisiert werden. Um den Entscheidungsprozess zu optimieren, müsse er kontinuierlich reflektiert und sowohl die Struktur des Tools als auch die Bewertungskriterien angepasst werden. Dies bringt neue Herausforderungen mit sich, denn das Tool sieht bisher bei einer Veränderung der Bewertungskriterien nicht zwangsläufig eine Neubewertung abgelehnter Ideen vor. Auf die Frage, ob die Flexibilität des Tools, z.B. Anpassung von Bewertungskriterien zulasten der Fairness gehe, antworteten die MA, die fehlende Passung zwischen Bewertungskriterien und den eingegebenen Ideen sei ein größeres Problem. Eine entscheidende Voraussetzung sei jedoch Transparenz: es sollte offen kommuniziert werden, wenn Kriterien verändert werden und $a b$ wann diese Änderung greift. Wichtig sei ebenfalls, dass diejenigen, die Ideen vorschlagen, die Möglichkeit bekommen, die Idee erneut einzureichen, wenn sie der Meinung sind, dass diese aufgrund der veränderten Bewertungskriterien besser passen würde.

Inwieweit das Ziel Effektivität erreicht wird, lässt sich nur ansatzweise beurteilen. Im Rahmen der Interviews wurden die Bewertungskriterien als hilfreich beschrieben, damit eine fundierte Umsetzungsentscheidung getroffen werden kann. Inwieweit die Kriterien die Umsetzung langfristig erfolgreicher Ideen unterstützen und Ideen mit geringer Erfolgswahrscheinlichkeit frühzeitig gestoppt werden, wird sich in der weiteren Erprobung des IdeaChecks zeigen.
Insgesamt berichteten die IT-MA im Interview motiviert zu sein, eigene Ideen über das Tool einzubringen und das Tool zu nutzen Die Ergebnisse der Expertenbefragung stützen diese Annahme (behavioral intention to use the system, $M=3,83, S D=0,72$ ). Insbesondere die Möglichkeit, eine Idee zu Ende zu denken und die Chance, dass diese nicht in Vergessenheit gerate, trage zur Motivation bei. Verstärkt wird diese durch die Möglichkeit, schnelles Feedback von anderen MA zu bekommen, wie diese zur Idee stehen. Negative, aber konstruktive Rückmeldung helfe dabei, die Idee zu optimieren, bevor sie zur Bewertung freigegeben wird. Die Möglichkeit zum Austausch untereinander und die gezielte Suche nach Unterstützern mit spezifischen Kompetenzen bzw. aus verschiedenen Bereichen könne die teamübergreifende Zusammenarbeit und das Teamklima positiv beeinflussen.

\section{Rahmenbedingungen für Innovationsfähigkeit und Einführung des Tools}

Das Tool stellt ein Werkzeug dar, bestehende Innovationsprozesse abzubilden und zu verändern. Die Einführung des Tools im Unternehmen ist aber kein Garant für einen funktionierenden Ideenfindungs- und Innovationsprozess im Unternehmen. Wichtige Einflussfaktoren sind die Organisationsstruktur und Unternehmenskultur sowie das Organisationsklima (Holz 2013).

Insbesondere bei der Ideengenerierung sind KMUs im Vergleich zu Großunternehmen durch ihre flacheren Hierarchien, größere Flexibilität und Anpassungsfähigkeit im Vorteil (Camisón-Zornoza et al. 2004). Allerdings nur unter der Voraussetzung, dass die Abteilungen eng zusammenarbeiten und nicht in Konkurrenz zueinander stehen (Maltz et al. 2001). Kurze Entscheidungswege fördern kreative Prozesse bei der Ideengenerierung (Damanpour 1991). Diese innovationsförderlichen Faktoren, wie abteilungsübergreifende Kooperationsmöglichkeiten bei der Ideenentwicklung und kurze Feedbackschleifen (z.B. schnelle Entscheidung darüber, ob eine Idee weitergeführt werden kann), sollten im Tool abgebildet und im Unternehmen etabliert werden. Die Schnelligkeit der Entscheidungen wird maßgeblich dadurch beeinflusst, wie viele Personen am Prozess beteiligt sind und wie viele Stufen bis zur Umsetzungsentscheidung durchlaufen werden. Um schnelle Entscheidungen treffen zu können, sollte die Anzahl der Stufen (Stages) und die Zahl der Entscheidungsträger so gering wie möglich gehalten werden.

Die Unternehmenskultur, als Grundgesamtheit gemeinsamer Werte, Normen und Einstellungen, die Entscheidung, Handlung und Einstellung der Beschäftigten prägt (Schein 1990), und die Art der Einführung des IdeaChecks spie- 
len eine entscheidende Rolle für die Akzeptanz und Nutzungsbereitschaft des Tools und letztendlich für den Erfolg von Innovationen (Hauschildt et al. 2016). Entscheidend ist, dass die Beschäftigten das Thema Innovation ,als wesentliches Element ihrer Kultur verstehen“ (Holz 2013, S. 310). Entsprechend muss Innovation im Unternehmen sowohl möglich als auch gewünscht sein. Voraussetzung dafür ist laut Aussage der IT-MA eine offensive Bewerbung des Tools, die nicht nur im Rahmen der Einführung, sondern laufend stattfinden sollte. Das Tool solle so präsent sein, dass alle MA es täglich nutzen können. Dazu gehöre, dass allen ihren im Tool beschriebenen Funktionen nachkämen. Beispielsweise sollten Gremienmitglieder ihre Aufgaben regelmäßig und zeitnah bearbeiten. Insbesondere zu Beginn von Veränderungsprozessen, wie bei der Tooleinführung, die durch Unsicherheit gekennzeichnet sind, kommt der Vorbildfunktion von Führungskräften eine wichtige Rolle zu (Chang et al. 2003). Eine innovationsfreundliche Unternehmenskultur zeichnet sich durch Aspekte transformationaler Führung aus, wie Unterstützung und das Lernen aus Fehlern (Abu El-Ella et al. 2013; Jung et al. 2003). Hinzukommen sichtbare Anreize (Cooper und Kleinschmidt 1994), z. B. durch die Integration der Ideenentwicklung in die persönlichen Jahresziele. Einfluss auf die persönlichen Ziele nehmen zu können, ist neben arbeitsbezogener Selbstwirksamkeit und Autonomie ein wichtiger Einflussfaktor, wenn es um das Einbringen von Ideen geht (Axtell et al. 2006; Hammond et al. 2011).

Der Aspekt des Organisationsklimas fokussiert auf Einstellungen, Motivation und Emotionen der Belegschaft (Holz 2013). MA in dem beschriebenen Fallbeispiel IT haben Vorbehalte gegen ein digitales Ideenmanagement, da frühere Einführungsversuche scheiterten. Es wurde der Wunsch geäußert, dass führungsseitig das Tool vorgestellt und sein Mehrwert gegenüber früheren Tools dargelegt werde. Weiterhin gaben die MA an, dass Unsicherheiten im Umgang mit dem Tool reduziert werden könnten, wenn das Tool anhand eines konkreten Beispiels eingeführt würde. Darüber hinaus trage die Funktion, andere Ideen einsehen zu können dazu bei, Unsicherheiten im Umgang mit dem Tool zu reduzieren. Als förderlich für die Motivation bewerteten die MA die Möglichkeit, an Ideen mitzuwirken, die sie selbst als sinnvoll und vielversprechend erachten. Ein zeitnahes wertschätzendes Feedback dient der Entwicklung eines innovationsförderlichen Klimas, bei dem MA eher bereit sind, sich am Innovationsprozess zu beteiligen. Das Einbringen eigener Innovationsideen stellt ein interpersonales Risiko dar, denn es besteht die Gefahr, dass die Idee abgelehnt wird. Eine wichtige Voraussetzung für Innovationsfähigkeit ist deshalb psychologische Sicherheit im Unternehmen, die sich dadurch auszeichnet, dass MA eher bereit, dieses Risiko einzugehen und ihre Ideen mitteilen (Edmondson und Lei 2014).
Zusammengefasst ergeben sich für die Einführung des Tools in Unternehmen die folgenden Handlungsempfehlungen: Das Tool sollte anhand eines konkreten Beispiels eingeführt und der Aufbau erklärt werden. Der Stellenwert von Innovation für das Unternehmen und die Verankerung in den Unternehmenszielen sollte verdeutlicht werden. Sinn, Zweck und Mehrwert des Tools sollten angesprochen werden. Den MA sollte ausreichend Zeit eingeräumt werden, das Tool zu explorieren und sich mit den Funktionen vertraut zu machen.

\section{Fazit und Ausblick}

Der IdeaCheck bietet die Möglichkeit, Ideen zu kommunizieren, weiterzuentwickeln und für Unternehmen nutzbar zu machen. Damit Ideen überhaupt erst entstehen und soweit aufgearbeitet werden, dass sie für andere nachvollziehbar kommuniziert werden können, spielen Organisationsstruktur, -kultur und -klima eine wichtige Rolle. Die Einführung eines digitalen Ideenmanagements eröffnet die Chance, Unternehmensstrukturen und -prozesse zu überdenken und zu verändern. Eine gelebte Innovationskultur ist entscheidend für den Erfolg: Innovation sollte ins Leitbild des Unternehmens aufgenommen werden, sodass MA sich mit dieser Kultur identifizieren können. Innovationsaktivitäten müssen in den Unternehmensalltag eingebunden werden. Schnelle wertschätzende Rückmeldung zu Ideen ist hierfür entscheidend. Das Tool unterstützt durch transparente und schnelle Feedbackschleifen während der Ausarbeitung der Ideen die Entstehung agiler Arbeitsmethoden, auch wenn der Stage Gate Prozess eine sequentielle Bearbeitung vorsieht und die Zusammenarbeit verteilt und primär digital vermittelt erfolgt. Eine offene, flexible und agile Innovationskultur lässt sich selbst in KMUs und vor allem in Großunternehmen nicht von heute auf morgen etablieren. Inwieweit dies durch das Tool unterstützt werden kann, ist eine spannende Frage für die künftige Forschung. Einfache Antworten liegen nicht auf der Hand, da das Tool einerseits die räumlich und zeitlich verteilte digitale Zusammenarbeit an Innovationen erleichtert, andererseits digital vermittelte Kommunikation den dafür hilfreichen Vertrauensaufbau erschwert. Für kleinere Unternehmen könnte dieses Dilemma leichter zu lösen sein als für Größere, da sich die Beschäftigten untereinander eher persönlich kennen und dadurch besser einschätzen können, wem sie ihr Vertrauen schenken. Um diese Fragen zu analysieren, könnte es fruchtbar sein, die bislang meist getrennten Forschungslinien zum individuellen Nutzungsverhalten neuer Technologien (Venkatesh et al. 2003), mit der Forschung zu digitaler Führung und Zusammenarbeit (Antoni und Syrek 2017) zu verbinden. 
Förderhinweis Das Verbundprojekt „Modelle ressourcenorientierter und effektiver Führung digitaler Projekt- und Teamarbeit (vLead)“, in dessen Rahmen das Teilprojekt „Digitale Projekt- und Teamarbeit leistungs- und ressourcenförderlich führen“ vom 01.04.2017-31.12.2020 durchgeführt wird, wird gefördert vom BMBF und dem Europäischen Sozialfonds unter dem Förderkennzeichen 02L15A080.

Danksagung Das vorgestellte Tool ist im Rahmen des vom Bundesministerium für Bildung und Forschung (BMBF) geförderten Forschungsprojekts „vLead - Modelle ressourcenorientierter und effektiver Führung bei digitaler Projekt- und Teamarbeit" entwickelt und erprobt worden.

Funding Open Access funding provided by Projekt DEAL.

Open Access Dieser Artikel wird unter der Creative Commons Namensnennung 4.0 International Lizenz veröffentlicht, welche die Nutzung, Vervielfältigung, Bearbeitung, Verbreitung und Wiedergabe in jeglichem Medium und Format erlaubt, sofern Sie den/die ursprünglichen Autor(en) und die Quelle ordnungsgemäß nennen, einen Link zur Creative Commons Lizenz beifügen und angeben, ob Änderungen vorgenommen wurden.

Die in diesem Artikel enthaltenen Bilder und sonstiges Drittmaterial unterliegen ebenfalls der genannten Creative Commons Lizenz, sofern sich aus der Abbildungslegende nichts anderes ergibt. Sofern das betreffende Material nicht unter der genannten Creative Commons Lizenz steht und die betreffende Handlung nicht nach gesetzlichen Vorschriften erlaubt ist, ist für die oben aufgeführten Weiterverwendungen des Materials die Einwilligung des jeweiligen Rechteinhabers einzuholen.

Weitere Details zur Lizenz entnehmen Sie bitte der Lizenzinformation auf http://creativecommons.org/licenses/by/4.0/deed.de.

\section{Literatur}

Akin, N., \& Rumpf, J. (2013). Führung virtueller Teams. Gruppendynamik und Organisationsberatung, 44(4), 373-387. https://doi. org/10.1007/s11612-013-0228-9.

Amabile, T.M., \& Pratt, M.G. (2016). The dynamic componential model of creativity and innovation in organizations: making progress, making meaning. Research in Organizational Behavior, 36, 157-183. https://doi.org/10.1016/j.riob.2016.10.001.

Antoni, C.H. (1987). Qualitätszirkel und Vorschlagswesen als Instrumente der Mitarbeiteraktivierung - Einsatzmöglichkeiten und Einsatzvoraussetzungen in der öffentlichen Verwaltung. In C. Böhret, H. Klages, H. Reinermann \& H. Siedentopf (Hrsg.), Herausforderungen an die Innovationskraft der Verwaltung (S. 397-422). Opladen: WDV. https://doi.org/10.1007/978-3322-83547-5 45.

Antoni, C.H., \& Ellwart, T. (2017). Informationsüberlastung bei digitaler Zusammenarbeit - Ursachen, Folgen und Interventionsmöglichkeiten. Gruppe. Interaktion. Organisation (GIO), 48, 305-315. https://doi.org/10.1007/s11612-017-0392-4

Antoni, C. H., \& Syrek, C. J. (2017). Digitalisierung der Arbeit: Konsequenzen für Führung und Zusammenarbeit. Gruppe. Interaktion. Organisation (GIO), 48, 247-258. https://doi.org/10.1007/ s11612-017-0391-5.

Anders, A. (2016). Team communication platforms and emergent social collaboration practices. International Journal of Business Communication, 53(2), 224-261. https://doi.org/10.1177/ 2329488415627273

Axtell, C., Holman, D., \& Wall, T. (2006). Promoting innovation: a change study. Journal of Occupational and Organiza- tional Psychology, 79(3), 509-516. https://doi.org/10.1348/ $096317905 \times 68240$

Bafoutsou, G., \& Mentzas, G. (2002). Review and functional classification of collaborational systems. International Journal of Information Management, 22, 281-305. https://doi.org/10.1016/ s0268-4012(02)00013-0.

Breuer, C., Hüffmeier, J., \& Hertel, G. (2017). Vertrauen per Mausklick: Wie Vertrauen in virtuellen Teams entstehen kann. Personal Quarterly, 69(2), 10-16.

Camisón-Zornoza, C., Lapiedra-Alcamí, R., Segarra-Ciprés, M., \& Boronat-Navarro, M. (2004). A meta-analysis of innovation and organizational size. Organization Studies, 25(3), 331-361. https:// doi.org/10.1177/0170840604040039.

Chang, A., Bordia, P., \& Duck, J. (2003). Punctuated equilibrium and linear progression: toward a new understanding of group development. Academy of Management Journal, 46(1), 106-117. https:// doi.org/10.5465/30040680.

Cooper, R.G. (2014). What's next?: after stage-gate. ResearchTechnology Management, 57(1), 20-31. https://doi.org/10.5437/ $08956308 \times 5606963$.

Cooper, R. G., \& Kleinschmidt, E. J. (1994). Determinants of timeliness in product development. Journal of Product Innovation Management, 11(5), 381-396. https://doi.org/10.1111/1540-5885. 1150381.

Damanpour, F. (1991). Organizational innovation: a meta-analysis of effects of determinants and moderators. Academy of Management Journal, 34(3), 555-590. https://doi.org/10.5465/256406.

Edmondson, A.C., \& Lei, Z. (2014). Psychological safety: the history, renaissance, and future of an interpersonal construct. Annual Review of Organizational Psychology and Organizational Behavior, 1(1), 23-43. https://doi.org/10.1146/annurev-orgpsych-03141309130.

El-Ella, A. N., Stoetzel, M., Bessant, J., \& Pinkwart, A. (2013). Accelerating high involvement: the role of new technologies in enabling employee participation in innovation. International Journal of Innovation Management, 17(6), 1340020. https://doi.org/10.1142/ s1363919613400203.

Eppler, M. J. \& Mengis, J. (2004) The concept of information overload: a review of literature from organization science, accounting, marketing, MIS, and related disciplines. The Information Society, 20(5), 325-344. https://doi.org/10.1080/01972240490507974

Frese, M., Kring, W., Soose, A., \& Zempel, J. (1996). Personal initiative at work: differences between East and West Germany. Academy of Management Journal, 39(1), 37-63. https://doi.org/10. 2307/256630.

Friedrich, P. (2014). Zielsteuerung von WLLB-Maßnahmen mithilfe der ALLWISS-Scorecard (ASC). In C.H. Antoni, P. Friedrich, A. Haunschild, M. Josten \& R. Meyer (Hrsg.), WorkLearnLife-Balance in der Wissensarbeit. Herausforderungen, Erfolgsfaktoren und Gestaltungshilfen für die betriebliche Praxis (S. 319-341). Berlin: Springer.

Garcia, R., \& Calantone, R. (2002). A critical look at technological innovation typology and innovativeness terminology: a literature review. Journal of Product Innovation Management, 19(2), 110-132. https://doi.org/10.1111/1540-5885.1920110.

Hammond, M.M., Neff, N.L., Farr, J.L., Schwall, A. R., \& Zhao, X. (2011). Predictors of individual-level innovation at work: a metaanalysis. Psychology of Aesthetics, Creativity, and the Arts, 5(1), 90-105. https://doi.org/10.1037/a0018556.

Handke, L., \& Kauffeld, S. (2019). Alles eine Frage der Zeit? Herausforderungen virtueller Teams und deren Bewältigung am Beispiel der Softwareentwicklung. Gruppe. Interaktion. Organisation. Zeitschrift für Angewandte Organisationspsychologie (GIO), 50(1), 33-41. https://doi.org/10.1007/s11612-019-00445-5.

Hauschildt, J., Salomo, S., Schultz, C., \& Kock, A. (2016). Innovationsmanagement, 6 . Auflage, München: Vahlen. 
Hegner, M. (2003). Methoden zur Evaluation von Software. Bd. 29. Bonn: Informationszentrum Sozialwissenschaften. https://nbnresolving.org/urn:nbn:de:0168-ssoar-50730-9

Highsmith, J.R. (2009). Agile project management: creating innovative products. London: Pearson Education.

Hockey, G. R. J. (1997). Compensatory control in the regulation of human performance under stress and high workload: a cognitiveenergetical framework. Biological Psychology, 45(1-3), 73-93. https://doi.org/10.1016/s0301-0511(96)05223-4

Hossain, E., Babar, M. A., \& Paik, H. (2009). Using Scrum in global software development: a systematic literature review. In Fourth IEEE International Conference on Global Software Engineering. https://doi.org/10.1109/icgse.2009.25.

Holz, M. (2013). Organisationsstruktur, Organisationskultur, Organisationsklima und Innovation. In D.E. Krause (Hrsg.), Kreativität, Innovation, Entrepreneurship (S. 305-319). https://doi.org/ 10.1007/978-3-658-02551-9_15.

Jørgensen, A.H. (1990). Thinking-aloud in user interface design: a method promoting cognitive ergonomics. Ergonomics, 33(4), 501-507. https://doi.org/10.1080/00140139008927157.

Jung, D. I., Chow, C., \& Wu, A. (2003). The role of transformational leadership in enhancing organizational innovation: hypotheses and some preliminary findings. The Leadership Quarterly, 14(4/5), 525-544. https://doi.org/10.1016/s1048-9843(03)00050$\mathrm{x}$.

Maltz, E., Souder, W.E., \& Kumar, A. (2001). Influencing R\&D/ marketing integration and the use of market information by R\&D managers: intended and unintended effects of managerial actions. Journal of Business Research, 52(1), 69-82. https://doi.org/10. 1016/s0148-2963(99)00096-x.

Möslein, K.M. (2009). Innovation als Treiber des Unternehmenserfolgs. In A. Zerfaß \& M. Möslein (Hrsg.), Kommunikation als Erfolgsfaktor im Innovationsmanagement (S. 3-21). Wiesbaden: Gabler.

Peris, M., \& Nüttgens, M. (2011). Anwendung der Unified Theory of Acceptance and Use of Technology zur Akzeptanzbestimmung von Web 2.0-Anwendungen in KMU-Netzwerken. In 6th Conference on Professional Knowledge Management-From Knowledge to Action. Bonn: Gesellschaft für Informatik e.V.

Rammer, C., Gottschalk, S., Peters, B., Bersch, J., \& Erdsiek, D. (2016). Die Rolle von KMU für Forschung und Innovation in Deutschland. Studien zum deutschen Innovationssystem, Bd. 102016. Berlin: Expertenkommission Forschung und Innovation. ZEW-Dokumentation

Reichwald, R. (2010). Innovation und Mitbestimmung - Chancen für den Hightech-Standort Deutschland. WSI, 2010(2), 62.

Ruef, M. (2002). String ties, weak ties and islands: structural and cultural predictors of organizational innovation. Industrial and Corporate Change, 11(3), 427-449. https://doi.org/10.1093/icc/11.3. 427.

Schein, E.H. (1990). Organizational culture. American Psychologist, 45(2), 109-119. https://doi.org/10.1037/0003-066x.45.2.109.

Spielkamp, A., \& Volkmann, C. (2005). Führungsinstrumente im Innovationsmanagement kleiner und mittlerer Unternehmen. In E. J. Schwarz und R. Harms (Hrsg.), Integriertes Ideenmanagement (S. 267-290). Wiesbaden: Deutscher Universitätsverlag. https://doi. org/10.1007/978-3-322-82153-9_14

Spielkamp, A., \& Rammer, C. (2006). Balanceact Innovation: Erfolgsfaktoren im Innovationsmanagement kleiner und mittlerer Unternehmen. Studien zum deutschen Innovationssystem, Bd. 06-2004. Berlin: Expertenkommission Forschung und Innovation. ZEWDokumentation

Spath, D., Heubach, D., \& Ardilio, A. (2010). Unterstützung des Innovationsmanagements durch IT-Systeme. HMD Praxis der Wirtschaftsinformatik, 47(3), 6-20. https://doi.org/10.1007/bf033 40470 .
Venkatesh, V., Morris, M.G., Davis, G. B., \& Davis, F. D. (2003). User acceptance of information technology: toward a unified view. MIS Quarterly, 27(3), 425. https://doi.org/10.2307/30036540.

von Bismarck, W.B. (2000). Das Vorschlagswesen: von der Mitarbeiteridee bis zur erfolgreichen Umsetzung. München: Rainer Hampp.

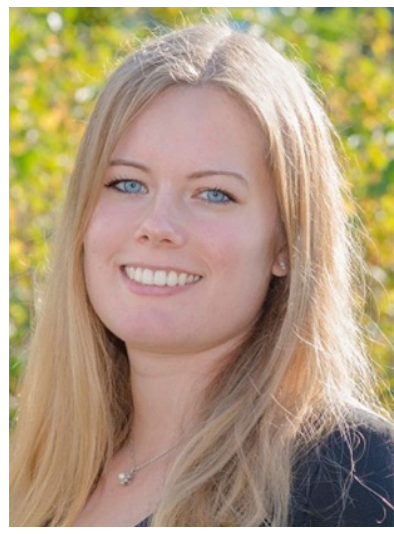

Anna T. Röltgen ist wissenschaftliche Mitarbeiterin in der Abteilung Arbeits-, Betriebs- und Organisationspsychologie an der Universität Trier. Sie ist im Forschungsprojekt „vLead“ tätig und beschäftigt sich in ihrer Promotion schwerpunktmäßig mit dem Austauschprozess zwischen Führungskraft und Mitarbeitern sowie dem Einfluss digitaler Medien auf Führungsprozesse und die Zusammenarbeit von Teams.

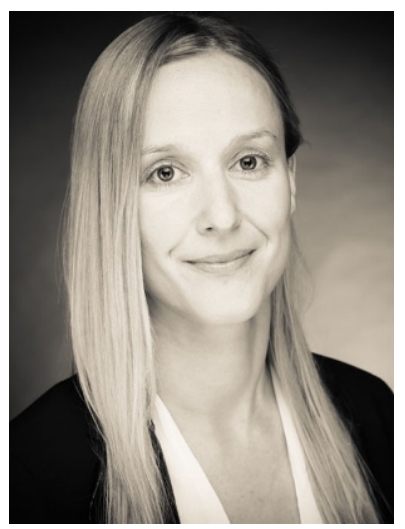

Dipl.-Psych. Valeria Bernardy ist wissenschaftliche Mitarbeiterin in der Abteilung Arbeits-, Betriebsund Organisationspsychologie an der Universität Trier. Sie ist im Forschungsprojekt ,vLead“ zu digitaler Zusammenarbeit in Teams tätig und beschäftigt sich in ihrer Promotion mit dem Einfluss individueller Faktoren auf die Teamarbeit und emergenten Teamprozessen.

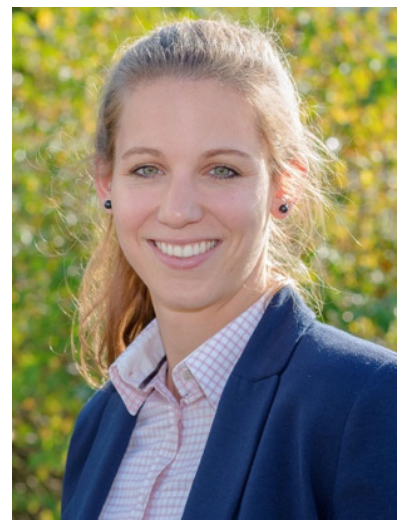

Rebecca Müller ist wissenschaftliche Mitarbeiterin in der Abteilung Arbeits-, Betriebs- und Organisationspsychologie an der Universität Trier. Sie ist im Forschungsprojekt „vLead“ tätig und beschäftigt sich in ihrer Promotion schwerpunktmäBig mit Teamprozessen in virtuellen Teams sowie dem Einfluss digitaler Medien auf geteilte mentale Modelle und die Zusammenarbeit von Teams. 


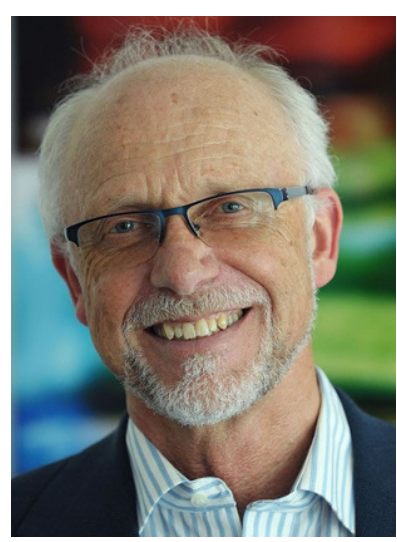

Prof. Dr. Conny H. Antoni ist

Professor für Arbeits-, Betriebsund Organisationspsychologie an

der Universität Trier. Seine Arbeitsschwerpunkte liegen in den Bereichen Teamarbeit, Führung, Stress und Work-Life-Balance, sowie Reward und Change Management. 